\title{
A Global Criterion for the Computation of Statistical Shape Model Parameters based on Correspondence Probabilities
}

\author{
Heike Hufnagel ${ }^{1,2}$, Xavier Pennec ${ }^{1}$, Jan Ehrhardt ${ }^{2}$, Nicholas Ayache ${ }^{1}$, \\ Heinz Handels ${ }^{2}$ \\ ${ }^{1}$ INRIA Asclepios Project, 06902 Sophia Antipolis, France \\ ${ }^{2}$ Medizinische Informatik, Universität Hamburg, 20246 Hamburg \\ h.hufnagel@uke.uni-hamburg.de
}

\begin{abstract}
A fundamental problem when building a statistical shape model (SSM) is the correspondence problem. We present an approach for unstructured point sets where one-to-one correspondences are replaced by correspondence probabilities between shapes which are determined using the Expectation Maximization - Iterative Closest Points registration. We propose a unified MAP framework to compute the model parameters which leads to an optimal adaption of the model to the observations. The optimization of the MAP explanation with respect to the observation and the generative model parameters leads to very efficient and closed-form solutions for (almost) all parameters. Experimental results on synthetic data and brain structures as well as a performance comparison with a statistical shape model built on one-to-one correspondences show the efficiency and advantages of this approach.
\end{abstract}

\section{Introduction}

In order to analyze shape variability it is necessary to determine correspondences between the observations of the training set. Several techniques were developed to automatically find exact correspondences [1], some in combination with the search for the SSM $[2,3]$ or a shape analysis [4]. An interesting approach proposes an entropy based criterion to find shape correspondences [5]. However, these methods are not easily adaptable to point sets as exact correspondences can only be determined between continuous surfaces, not between point cloud representations of surfaces. The SoftAssign algorithm tries to solve this problem with an initial probabilistic formulation [6]. In order to build a SSM for unstructured point sets, we advance the probabilistic concept of [7] using the EM-ICP registration which proved to be robust, precise, and fast [8]. The approach solves for the mean shape and the eigenmodes in a unique criterion without the need of one-to-one correspondences as is usually required by the PCA. This article focuses on the validation of the work presented in [9]. 


\section{Methods and Experiments}

We first realize a MAP estimation of the model and observation parameters which leads to a unique criterion. We then compute the SSM which best fits the given data set by optimizing the global criterion iteratively with respect to all model and observation parameters. A key part of our method is that we can find a closed-form solution for nearly each of the parameters.

\subsection{Statistical Shape Model Built on Correspondence Probabilities}

In the process of computing the SSM, we distinguish strictly between model parameters and observation parameters. The generative SSM is explicitly defined by 4 model parameters: The mean shape $\bar{M} \in \mathbb{R}^{3 N_{m}}$ parameterized by $N_{m}$ points $m_{j} \in \mathbb{R}^{3}$, the eigenmodes $v_{p}$ consisting of $N_{m}$ 3D vectors $v_{p j}$, the associated standard deviations $\lambda_{p}$ which describe the impact of the eigenmodes, and the number $n$ of eigenmodes. Using the generative model $\Theta=\left\{\bar{M}, v_{p}, \lambda_{p}, n\right\}$ of a given structure, the shape variations of that structure can be generated by $M_{k}=\bar{M}+\sum_{p=1}^{n} \omega_{k p} v_{p}$ with $\omega_{k p} \in \mathbb{R}$ being the deformation coefficients. The shape variations along the modes follow a Gaussian probability with variance $\lambda_{p}$ and $\Omega_{k}=\left\{\omega_{k p}, p=0, \ldots, n-1\right\}$.

$$
p\left(M_{k} \mid \Theta\right)=p\left(\Omega_{k} \mid \Theta\right)=\prod_{p=1}^{n} p\left(\omega_{k p} \mid \Theta\right)=\frac{1}{(2 \pi)^{n / 2} \prod_{p=1}^{n} \lambda_{p}} \exp \left(-\sum_{p=1}^{n} \frac{\omega_{k p}^{2}}{2 \lambda_{p}^{2}}\right)
$$

In order to account for the unknown position and orientation of the model in space, we introduce the linear transformation $T_{k}$. A model point $m_{j}$ can then be deformed and placed in space by the linear transformation $T_{k}$ with $T_{k}$ *

$m_{k j}=T_{k} \star\left(\bar{m}_{j}+\sum_{p} \omega_{k p} v_{p}\right)$. Finally, we model each observation point $s_{k i}$ as a Gaussian measurement of a (transformed) model point $m_{j}$. As we do not know the originating model point for each observation point $s_{k i}$, the probability of a given $s_{k i}$ is described by a Mixture of Gaussians and the probability for the whole scene $S_{k}$ becomes:

$$
p\left(S_{k} \mid M, T_{k}\right)=\prod_{i=1}^{N_{k}} \frac{1}{N_{m}} \sum_{j=1}^{N_{m}} p\left(s_{k i} \mid m_{j}, T_{k}\right)
$$

with $p\left(s_{k i} \mid m_{j}, T_{k}\right)=(2 \pi)^{-3 / 2} \sigma^{-1} \exp \left(-\frac{1}{2 \sigma^{2}}\left(s_{k i}-T_{k} \star m_{j}\right)^{T} .\left(s_{k i}-T_{k} \star m_{j}\right)\right)$. We summarize the observation parameters as $Q_{k}=\left\{\Omega_{k}, T_{k}\right\}$.

\subsection{Derivation of the Global Criterion Using a MAP Approach}

When building the SSM, we use $N$ observations $S_{k} \in \mathbb{R}^{3 N_{k}}$, and we are interested in the parameters linked to the observations $Q=\left\{Q_{k}\right\}$ as well as the unknown 
model parameters $\Theta$. In order to determine all parameters of interest, we optimize a MAP on $Q$ and $\Theta$.

$$
\operatorname{MAP}=-\sum_{k=1}^{N} \log \left(p\left(Q_{k}, \Theta \mid S_{k}\right)\right)=-\sum_{k=1}^{N} \log \left(\frac{p\left(S_{k} \mid Q_{k}, \Theta\right) p\left(Q_{k} \mid \Theta\right) p(\Theta)}{p\left(S_{k}\right)}\right)
$$

As $p\left(S_{k}\right)$ does not depend on $\Theta$ and $p(\Theta)$ is assumed to be uniform, the global criterion integrating our unified framework is the following:

$$
C(Q, \Theta)=-\sum_{k=1}^{N}\left(\log \left(p\left(S_{k} \mid Q_{k}, \Theta\right)\right)+\log \left(p\left(Q_{k} \mid \Theta\right)\right)\right)
$$

The first term describes the ML criterion (eq. (2)) whereas the second term is the prior on the deformation coefficients $\omega_{k p}$ as described in eq.(1). Dropping the constants, our criterion simplifies to $C(Q, \Theta) \sim \sum_{k=1}^{N} C_{k}\left(Q_{k}, \Theta\right)$ with

$$
C_{k}\left(Q_{k}, \Theta\right)=\sum_{p=1}^{n}\left(\log \left(\lambda_{p}\right)+\frac{\omega_{k p}^{2}}{2 \lambda_{p}^{2}}\right)-\sum_{i=1}^{N_{k}} \log \left(\sum_{j=1}^{N_{m}} \exp \left(-\frac{\left\|s_{k i}-T_{k} \star m_{k j}\right\|^{2}}{2 \sigma^{2}}\right)\right)(5)
$$

This equation is the heart of the unified framework for the model computation and its fitting to observations. By optimizing it alternately with respect to the operands in $\{Q, \Theta\}$, we are able to determine all parameters we are interested in. In a first step, all observations are aligned with the initial mean shape by estimating the $T_{k}$ using the EM-ICP. In order to robustify, we used a multi-scaling scheme concerning the variance $\sigma^{2}$, for details please refer to [9]. Starting from the initial model parameters $\Theta$, we then fit the model to each of the observations. Next, we fix the observation parameters $Q_{k}$ and update the model parameters. This is iterated until convergence.

\section{$2.3 \quad$ Experiments}

We present two experiments we ran in order to evaluate our approach. A typical correspondence problem: One of our arguments against the concept of enforcing homologies between points representing surfaces is the fact that certain shape structures of one observation might not appear on all observations of the training set. We constructed a synthetic data set containing 20 observations in order to illustrate this problem, see figure 1 a). Half of the ellipsoids are equipped with a bump, and the other half are not. A reliable SSM should be able to represent both classes by including this distinction in its variability model. In order to compare the results, we generated two SSMs, one by using our new method, the other one by using the traditional ICP and PCA.

Our data set consists of $N=21$ left segmented putamen observations (approximately $20 \mathrm{~mm} \times 20 \mathrm{~mm} \times 40 \mathrm{~mm}$ ) which are represented by min 994 and $\max 1673$ points (Fig. 1c)). In order to assess the quality of a SSM, we measure two performance measures as proposed in [10]. A good generalization ability is 
Table 1. Performance Measures. Shape distances found in generalization experiments (7 leave-one-out tests) and in specificity experiments (500 random shapes) with our SSM approach and with an ICP+PCA approach using 18 eigenmodes

\begin{tabular}{lcc}
\hline Generalization ability & ICP+PCA model & our SSM \\
\hline average mean distance + standard deviation in $\mathrm{mm}$ & $0.610 \pm 0.089$ & $0.447 \pm 0.101$ \\
average max. distance + standard deviation in $\mathrm{mm}$ & $4.388 \pm 0.930$ & $2.426 \pm 0.712$ \\
\hline Specificity & ICP+PCA model & our SSM \\
\hline average mean distance + standard deviation in mm & $0.515 \pm 0.117$ & $0.452 \pm 0.020$ \\
\hline
\end{tabular}

important for recognition purposes as a SSM must be able to adopt the shape of an unseen observation which comes from the same structure type. We test it in a series of leave-one-out experiments. The SSM is first aligned with the unseen observation, then the matching is optimized by finding the best deformation coefficients $\omega$. Finally, the distance of the deformed SSM to the left-out observation is measured. The specificity of a SSM must be high for shape prediction purposes as the SSM should only adopt shapes similar to the ones in the underlying training set. We generated random shapes $x$ which are uniformly distributed with $\sigma$ being equal to the standard deviation of the SSM. Next, we computed the distance of the random shapes to the closest observations in the training data set. As we do not have surface representations, we chose a distance measure which computes the average minimum point distance between the deformed model points and the observation points.

\section{Results}

For our SSM, the following parameters were chosen: $\sigma_{\text {start }}=4 \mathrm{~mm}$, reduction factor $=0.85,10$ iterations (EM-ICP) with 5 SSM iterations. For the ICP+PCA $\mathrm{SSM}$, we iterated the ICP 50 times. The results for the experiments concerning the typical correspondence problem are shown in Figure 1 a),b). For both meth-

a)

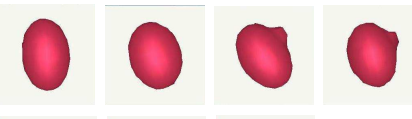

b)

c)

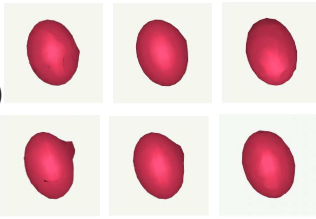

d)

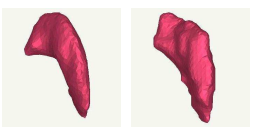

e) rico

Fig. 1. SSM on synthetic data set: (a) Ellipsoid observation examples; (b) Results SSM/ICP; (c) Results SSM/EM-ICP with final mean shape (middle), and the mean shapes deformed with respect to the first eigenmode $\left(\bar{M}-3 \lambda_{1} \boldsymbol{v}_{1}\right.$ and $\left.\bar{M}+3 \lambda_{1} \boldsymbol{v}_{1}\right)$; (d) Putamen observation examples; (e) Exact correspondence versus correspondence probabilities. Left: ICP, right: EM-ICP. 
ods, we chose the initial model from the ellipsoid class without bump. The first row shows the mean shape and eigenmodes for the SSM built by the ICP+PCA SSM whereas the second row shows those for our SSM.

The results of the testing series for the generalization ability and the specificity for both our SSM and the ICP+PCA SSM on putamen data are depicted in table 1 . We performed the leave-one out test for 7 different unknown observations. The specificity was computed for 500 random shapes. We computed the average and maximal distances as well as the variances.

\section{Discussion}

We proposed a mathematically sound and unified framework for the computation of model parameters and observation parameters and succeeded in determining a closed form solution for optimizing the associated criterion alternately for all parameters. Experiments showed that our algorithm works well and leads to plausible results. It seems to be robust to different initial mean shape choices and is stable even for small numbers of observations. We showed the efficiency of our approach compared with a SSM built by the traditional ICP and PCA for a typical correspondence problem on synthetic data: The SSM based on the EMICP models the whole data set, it is able to represent the ellipsoids featuring a bump and those without as that deformation information is included in its variability model. On the other hand, the results show that the SSM based on the ICP is not able to model the bump. This is due to the fact that the ICP only takes into account the closest point when searching for correspondence, thus, the point on top of the bump is not involved in the registration process. The EM-ICP, however, evaluates the correspondence probability of all points, therefore, also the point on top of the bump is matched. We illustrated these two concepts in figure 1d). Furthermore, in the test series on putamen data, our SSM achieved superior results in both performance measures. Especially the values of the maximal distance illustrate the benefit of the new approach. From a theoretical point of view, a very powerful feature of our method is that we are optimizing a unique criterion. Thus, the convergence is ensured.

Acknowledgement. This work is supported by a grant from the DFG, HA2355.

\section{References}

1. Besl PJ, McKay ND. A method for registration of 3D shapes. IEEE Trans Pattern Anal Mach Intell. 1992;14:239-56.

2. Davies RH, Twining CJ, Cootes TF. A minimum description length approach to statistical shape modeling. IEEE Medical Imaging. 2002;21(5):525-37.

3. Heimann $\mathrm{T}$, Wolf I, Williams $\mathrm{T}$, et al. 3D Active shape models using gradient descent optimization of description length. Proc IPMI. 2005;3565:566-77.

4. Tsai A, Wells WM, Warfield SK, et al. An EM algorithm for shape classification based on level sets. Med Image Anal. 2005;9:491-502. 
5. Cates J, Meyer M, Fletcher PT, et al. Entropy-based particle systems for shape correspondences. Proc MICCAI. 2006; p. 90-9.

6. Rangarajan A, Chui H, Bookstein FL. The softassign procrustes matching algorithm. Proc IPMI. 1997;1230:29-42.

7. Hufnagel H, Pennec X, Ehrhardt J, et al. Point-based statistical shape models with probabilistic correspondences and affine EM-ICP. Proc BVM. 2007; p. 434-8.

8. Granger S, Pennec X. Multi-scale EM-ICP: A fast and robust approach for surface registration. Lec Not Comp Sci. 2002;2525:418-32.

9. Hufnagel H, Pennec X, Ehrhardt J, et al. Shape analysis using a point-based statistical shape model built on correspondence probabilities. Proc MICCAI. 2007;1:95967 .

10. Styner M, Rajamani KT, Nolte LP, et al. Evaluation of 3D correspondence methods for model building. Proc IPMI. 2003;2732:63-75. 